EQUIPE EDITORIAL E AVALIADORES AD HOC ${ }^{1}$

\begin{abstract}
EDITORA-CHEFE
Dra. Greice da Silva Lorenzzetti Andreis

Instituto Federal de Educação, Ciência e Tecnologia do Rio Grande do Sul (IFRS), Campus Caxias do Sul, Caxias do Sul, RS, Brasil

\section{EDITORA ADJUNTA}

Dra. Katia Arcaro

Instituto Federal de Educação, Ciência e Tecnologia do Rio Grande do Sul (IFRS), Campus Caxias do Sul, Caxias do Sul, RS, Brasil
\end{abstract}

\title{
EDITOR EXECUTIVO
}

Esp. David Matos Milhomens

Instituto Federal de Educação, Ciência e Tecnologia de Santa Catarina (IFSC), Campus Florianópolis, Florianópolis, SC, Brasil

\section{COMISSÃO EDITORIAL}

Dra. Daiane Scopel Boff

Instituto Federal de Educação, Ciência e Tecnologia do Rio Grande do Sul (IFRS), Campus Caxias do Sul, Caxias do Sul, RS, Brasil

\section{Dra. Elisângela Pinto Francisquetti}

Instituto Federal de Educação, Ciência e Tecnologia do Rio Grande do Sul (IFRS), Campus Feliz, Feliz, RS, Brasil

Dra. Greice da Silva Lorenzzetti Andreis

Instituto Federal de Educação, Ciência e Tecnologia do Rio Grande do Sul (IFRS), Campus Caxias do Sul, Caxias do Sul, RS, Brasil

Dr. Henri Luiz Fuchs

Instituto Federal de Educação, Ciência e Tecnologia do Rio Grande do Sul (IFRS), Campus Bento Gonçalves, Bento

Gonçalves, RS, Brasil

\footnotetext{
${ }^{1}$ Como citar: ANDREIS, Greice da Silva Lorenzzetti. Equipe Editorial e Avaliadores Ad hoc. REMAT:
}

Revista Eletrônica da Matemática, Bento Gonçalves, RS, v. 7, n. 1, e1002, 30 jun. 2021. DOI:

https://doi.org/10.35819/remat2021v7i1id5284. 
Me. Jeremias Stein Rodriguês

Instituto Federal de Educação, Ciência e Tecnologia de Santa Catarina (IFSC), Campus Florianópolis, Florianópolis, SC, Brasil

\title{
Dra. Katia Arcaro
}

Instituto Federal de Educação, Ciência e Tecnologia do Rio Grande do Sul (IFRS), Campus Caxias do Sul, Caxias do Sul, RS, Brasil

Me. Michelsch João da Silva

Instituto Federal de Educação, Ciência e Tecnologia de Santa Catarina (IFSC), Campus Florianópolis, Florianópolis, SC, Brasil

\section{Dr. Rafael Cavalheiro}

Universidade Federal do Rio Grande (FURG), Campus Santo Antônio da Patrulha, Santo Antônio da Patrulha, RS, Brasil

\section{Dr. Rene Carlos Cardoso Baltazar Júnior}

Universidade Federal do Rio Grande (FURG), Campus Santo Antônio da Patrulha, Santo Antônio da Patrulha, RS, Brasil

\section{CONSELHO EDITORIAL CONSULTIVO}

\author{
Dra. Adriana Breda \\ Universitat de Barcelona, Barcelona, Espanha
}

Dra. Andriceli Richit

Instituto Federal de Educação, Ciência e Tecnologia Catarinense (IFC), Campus Concórdia, Concórdia, SC, Brasil

\section{Dr. Carloney Alves de Oliveira}

Universidade Federal de Alagoas (UFAL), Centro de Educação (CEDU), Campus Aristóteles Calazans Simões, Maceió, AL, Brasil

\section{Dra. Carolina Augusta Assumpção Gouveia}

Centro Universitário de Valença (UNIFAA), Valença, RJ, Brasil

\section{Me. Dailson Evangelista Costa}

Universidade Federal do Tocantins, Campus Universitário de Arraias, Arraias, TO, Brasil

\section{Dra. Franciele Corti}

Universitat de Barcelona, Barcelona, Espanha

\section{Dra. Maité Kulesza}

Universidade Federal Rural de Pernambuco (UFRPE), Departamento de Matemática, Recife, PE, Brasil

\section{Dr. Moiseis Cecconello}

Universidade Federal de Mato Grosso (UFMT), Cuiabá, MT, Brasil

\author{
Dr. Rafayel Teymurazyan \\ Universidade de Coimbra, Coimbra, Portugal \\ Dr. Wagner Marcelo Pommer \\ Universidade Federal de São Paulo (UNIFESP), Campus Diadema, Diadema, SP, Brasil
}




\section{REVISORES DE TEXTO}

Grad. Adriano Ernesto Trindade

Universidade Federal do Pampa (UNIPAMPA), RS, Brasil

Ma. Andréa de Araújo Rubert

Universidade do Minho (UMINHO), Portugal

Esp. Ângela Marta Emídio

Universidade Paulista (UNIP), SP, Brasil

Grad. Bruna Santiago dos Reis

Universidade Federal do Rio Grande do Sul (UFRGS), Porto Alegre, RS, Brasil

Grad. Bruno Scienza Schmidt

Universidade do Vale do Rio dos Sinos (UNISINOS), São Leopoldo, RS, Brasil

Ma. Camila Gabriele da Cruz Clemente

Universidade Federal de São Carlos (UFSCar), São Carlo, SP, Brasil

Dra. Caroline de Morais

Instituto Federal de Educação, Ciência e Tecnologia do Rio Grande do Sul (IFRS), Campus Vacaria, Vacaria, RS, Brasil

Esp. Claudia Moraes Dal Molin

Faculdade Unyleya, Rio de Janeiro, RJ, Brasil

Dr. Claudionor Ferreira Araújo

Instituto Federal de Educação, Ciência e Tecnologia de Alagoas (IFAL), Campus Maceió, Maceió, AL, Brasil

\section{Esp. Débora dos Santos Godoi Mariano}

Instituto Federal de Educação, Ciência e Tecnologia Fluminense (IFF), Campus Macaé, RJ, Brasil Serviço Social da Indústria no Estado do Rio de Janeiro (SESI), Escola Firjan SESI, Macaé, RJ, Brasil

\section{Esp. Denise Escher}

São Paulo, SP, Brasil

Me. Diego Ravarotto da Costa

Universidade Federal do Rio Grande (FURG), Rio Grande, RS, Brasil

Grad. Fabiana Santos Oliveira

GO, Brasil

Dra. Fernanda Delatorre

Universidade Federal de Santa Catarina (UFSC), Campus Florianópolis, Florianópolis, SC, Brasil

Ma. Fernanda Gonçalves Vieira

Universidade Federal do Rio Grande (FURG), Rio Grande, RS, Brasil

Secretaria Municipal de Educação e Desporto de Pelotas (SMED), Pelotas, RS, Brasil

Grad. Gabriel Salinet Rodrigues

Universidade Federal de Santa Maria (UFSM), Santa Maria, RS, Brasil 


\section{Esp. Ivone Teixeira \\ Petrópolis, RJ, Brasil}

Esp. Jailson Luiz Jablonski

Secretaria de Educação do Estado do Rio Grande do Sul (SEDUC), Porto Alegre, RS, Brasil

Faculdade Educacional da Lapa (FAEL), Brasil

Faculdade do Leste Mineiro (FACULESTE), Brasil

Grad. Joana Restelli Ferla

Universidade do Vale do Rio dos Sinos (UNISINOS), São Leopoldo, RS, Brasil

Ma. Karina dos Reis Costantin

Universidade Federal de Santa Maria (UFSM), Santa Maria, RS, Brasil

Ma. Karine Mathias Döll

Universidade Federal do Rio Grande do Sul (UFRGS), Porto Alegre, RS, Brasil

Ma. Manuela Damiani Poletti da Silva

Instituto Federal de Educação, Ciência e Tecnologia do Rio Grande do Sul (IFRS), Campus Caxias do Sul, Caxias do Sul, RS, Brasil

Me. Marden Oliveira Silva

Centro Federal de Educação Tecnológica de Minas Gerais (CEFET-MG), Belo Horizonte, MG, Brasil

Ma. Maria Estela Magalhães Reis

Universidade Presbiteriana Mackenzie, São Paulo, SP, Brasil

Dra. Maria Rodrigues de Oliveira

São Paulo, SP, Brasil

Me. Maurício Marques Sortica

Universidade Federal de Uberlândia (UFU), Uberlândia, MG, Brasil

Esp. Nathalia Santos Borges

Centro Universitário Fibra (FIBRA), Belém, PA, Brasil

Ma. Nathalie de Souza Kappke

Universidade Federal do Rio Grande do Sul (UFRGS), Porto Alegre, Brasil

Esp. Patrícia Guimarães Pereira

Universidade Federal do Oeste do Pará (UFOPA), Santarém, PA, Brasil

Dra. Raquel Salcedo Gomes

Universidade Federal do Rio Grande do Sul (UFRGS), Campus Litoral Norte, Tramandaí, RS, Brasil

Me. Roberto Rossi Menegotto

Universidade de Caxias do Sul (UCS), Caxias do Sul, RS, Brasil

Dra. Simone Xavier Moreira

Instituto Federal de Educação, Ciência e Tecnologia Sul-rio-grandense (IFSul), Campus Camaquã, RS, Brasil 
Ma. Verônica Wegner

Instituto Federal de Educação, Ciência e Tecnologia do Rio Grande do Sul (IFRS), Campus Sertão, Sertão, RS, Brasil

\section{APOIO TÉCNICO}

Srta. Glauciane Klein Burgiert Padilha

Instituto Federal de Educação, Ciência e Tecnologia do Rio Grande do Sul (IFRS), Bolsista BICTES/IFRS, Campus Caxias do Sul, Caxias do Sul, RS, Brasil

\section{AVALIADORES AD HOC}

Me. Adalberto Ayjara Dornelles Filho

Fundação Instituto Brasileiro de Geografia e Estatística (IBGE), Porto Alegre, RS, Brasil

\section{Dr. Adriano Fonseca}

Universidade Federal do Norte do Tocantins (UFNT), Campus Universitário de Araguaína, Araguaína, TO, Brasil

\section{Dr. Adriano Rodrigues de Melo}

Instituto Federal de Educação, Ciência e Tecnologia Catarinense (IFC), Campus Araquari, Araquari, SC, Brasil

\section{Dr. Adriano Vitor}

Instituto Federal de Educação, Ciência e Tecnologia de Santa Catarina (IFSC), Campus Florianópolis, Florianópolis, SC, Brasil

\section{Dr. Álvaro Krüger Ramos}

Universidade Federal do Rio Grande do Sul (UFRGS), Campus do Vale, Porto Alegre, RS, Brasil

\section{Dra. Ana Carolina Costa Pereira}

Universidade Estadual do Ceará (UECE), Fortaleza, CE, Brasil

Dra. Andréa Pavan Perin

Serviço Social da Indústria (SESI), SP, Brasil

Faculdade de Tecnologia de Itapetininga (FATEC), Itapetininga, SP, Brasil

Me. Antonio Carlos Mometti

Universidade de São Paulo (USP), Faculdade de Educação, São Paulo, SP, Brasil

\section{Dr. Arlane Manoel Silva Vieira}

Universidade Federal do Maranhão (UFMA), Campus Universitário de Codó, Codó, MA, Brasil

\section{Dr. Augusto Vieira Cardona}

Pontifícia Universidade Católica do Rio Grande do Sul (PUCRS), Porto Alegre, RS, Brasil

Me. Brehme D'napoli Reis de Mesquita

Instituto Federal de Educação, Ciência e Tecnologia do Maranhão (IFMA), Campus Açailândia, Açailândia, MA, Brasil

\section{Me. Carlos Bino de Souza}

Instituto Federal de Educação, Ciência e Tecnologia de Pernambuco (IFPE), Campus Pesqueira, Pesqueira, PE, Brasil 
Me. Carlos Vitor da Silva Sarmento

Universidade Federal de Pernambuco (UFPE), Recife, PE, Brasil

Dra. Carolina Noele Renz

Fundação Universidade Federal de Ciências da Saúde de Porto Alegre (UFCSPA), Porto Alegre, RS, Brasil

Ma. Cintia Schneider

Universidade Federal de Santa Catarina (UFSC), Programa de Pós-Graduação em Educação Científica e Tecnológica, SC, Brasil

$$
\begin{aligned}
& \text { Dr. Claus Haetinger } \\
& \text { Lajeado, RS, Brasil }
\end{aligned}
$$

Dr. Dagoberto Adriano Rizzotto Justo

Universidade Federal do Rio Grande do Sul (UFRGS), Porto Alegre, RS, Brasil

\section{Dra. Danielle Santos Azevedo}

Instituto Federal de Educação, Ciência e Tecnologia do Rio Grande do Sul (IFRS), Campus Alvorada, Alvorada, RS, Brasil

\section{Dr. David Antonio da Costa}

Universidade Federal de Santa Catarina (UFSC), Florianópolis, SC, Brasil

Dra. Débora de Lima Velho Junges

Instituto Federal de Educação, Ciência e Tecnologia Catarinense (IFC), Campus Fraiburgo, Fraiburgo, SC, Brasil

\section{Dr. Edberto Ferneda}

Universidade Estadual Paulista Júlio de Mesquita Filho (UNESP), Faculdade de Filosofia e Ciências, Campus de Marília, Marília, SP, Brasil

\section{Dr. Eudes Antonio da Costa}

Universidade Federal do Tocantins (UFT), Campus Arraias, Arraias, TO, Brasil

\section{Dr. Fabiano Carlos Cidral}

Instituto Federal de Educação, Ciência e Tecnologia de Santa Catarina (IFSC), Campus Florianópolis, Florianópolis, SC, Brasil

Dra. Fernanda Vital de Paula

Universidade Federal do Norte do Tocantins (UFNT), Araguaína, TO, Brasil

\section{Dra. Franciele Corti}

Universitat de Barcelona, Barcelona, Espanha

Dra. Francieli Aparecida Vaz

Universidade Federal do Pampa (UNIPAMPA), Bagé, RS, Brasil

Me. Guilherme Wagner

Rede Municipal de Educação de Florianópolis (REMED/FLORIPA), Florianópolis, SC, Brasil Universidade Federal de Santa Catarina (UFSC), Florianópolis, SC, Brasil

Dr. Idemar Vizolli

Universidade Federal do Tocantins (UFT), Palmas, TO, Brasil 


\title{
Me. Igor Antonio de Oliveira Carvalho
}

Instituto Federal de Educação, Ciência e Tecnologia do Maranhão (IFMA), Campus Timon, Timon, MA, Brasil

\author{
Ma. Ivonne Coromoto Sánchez \\ Universidade Federal do Pará (UFPA), Belém, PA, Brasil
}

Me. Jackson Luís Santos de Vargas

Secretaria da Educação do Estado do Rio Grande do Sul (SEDUC), Guaíba, RS, Brasil

Me. Jeremias Stein Rodriguês

Instituto Federal de Educação, Ciência e Tecnologia de Santa Catarina (IFSC), Campus Florianópolis, Florianópolis, SC, Brasil

\section{Dr. João Cândido Moraes Neves}

Instituto Federal de Educação, Ciência e Tecnologia do Rio Grande do Sul (IFRS), Campus Caxias do Sul, Caxias do Sul, RS, Brasil

\section{Dr. João Carlos Pereira de Moraes}

Universidade Federal do Pampa (UNIPAMPA), Jaguarão, RS, Brasil

Me. João Paulo da Cruz Almeida

Prefeitura Municipal de Castilho, Castilho, SP, Brasil

\section{Dr. João Vitor Teodoro}

Universidade Federal do Triângulo Mineiro (UFTM), Campus Iturama, Iturama, MG, Brasil

$$
\text { Dr. José Luiz de Souza Pio }
$$

Universidade Federal do Amazonas (UFAM), Instituto de Computação, Manaus, AM, Brasil

\section{Dr. José Sávio Bicho de Oliveira}

Universidade Federal do Sul e Sudeste do Pará (UNIFESSPA), Faculdade de Educação do Campo, Marabá, PA, Brasil

$$
\text { Dra. Juliana Sartori Ziebell }
$$

Universidade Federal do Rio Grande do Sul (UFRGS), Instituto de Matemática e Estatística, Campus do Vale, Porto Alegre, RS, Brasil

Me. Kévin Allan Sales Rodrigues

Universidade de São Paulo (USP), São Paulo, SP, Brasil

\section{Dr. Lênio Fernandes Levy}

Universidade Federal do Pará (UFPA), Instituto de Ciências Exatas e Naturais, Campus Universitário do Guamá, Belém, PA, Brasil

\section{Ma. Luana Henrichsen}

Universidade Regional do Noroeste do Estado do Rio Grande do Sul (UNIJUí), Campus ljuí, RS, Brasil

\section{Dr. Lucas Henrique Backes}

Universidade Federal do Rio Grande do Sul (UFRGS), Porto Alegre, RS, Brasil

Dra. Luciana Santos da Silva Martino

Colégio Pedro II (CPII), Rio de Janeiro, RJ, Brasil 


\title{
Dr. Luciano Nascimento
}

Universidade Federal de Pernambuco (UFPE), Recife, PE, Brasil

\author{
Me. Luis Andres Castillo Bracho \\ Universidade Federal do Pará (UFPA), Belém, PA, Brasil
}

Dr. Marcelo Sobottka

Universidade Federal de Santa Catarina (UFSC), Centro de Física e Matemática, Departamento de Matemática, Campus Trindade, Florianópolis, SC, Brasil

Dra. Márcia Jussara Hepp Rehfeldt

Universidade do Vale do Taquari (UNIVATES), Lajeado, RS, Brasil

Dra. Maria Auxiliadora Bueno Andrade Megid

Pontifícia Universidade Católica de Campinas (PUC-CAMPINAS), Programa de Pós-Graduação em Educação, Campinas, SP, Brasil

Dra. Maria do Carmo Pacheco de Toledo Costa

Universidade Federal de Lavras (UFLA), Departamento de Matemática e Matemática Aplicada, Lavras, MG, Brasil

Dra. Marília Luiza Matte

Colégio Militar de Porto Alegre (CMPA), Porto Alegre, RS, Brasil

Dra. Miriam Telichevesky

Universidade Federal do Rio Grande do Sul (UFRGS), Instituto de Matemática, Porto Alegre, RS, Brasil

Dr. Moisés Aparecido do Nascimento

Universidade Tecnológica Federal do Paraná (UTFPR), Campus Pato Branco, Pato Branco, PR, Brasil

Dra. Naíma Soltau Ferrão

Instituto Federal de Educação, Ciência e Tecnologia do Sul de Minas Gerais (IFSULDEMINAS), Campus Machado, Machado, MG, Brasil

Dr. Nelson Machado Barbosa

Universidade Estadual do Norte Fluminense Darcy Ribeiro (UENF), Campos dos Goytacazes, RJ, Brasil

Dra. Neyva Maria Lopes Romeiro

Universidade Estadual de Londrina (UEL), Centro de Ciências Exatas, Departamento de Matemática, Londrina, PR, Brasil

\section{Dr. Nicolau Matiel Lunardi Diehl}

Instituto Federal de Educação, Ciência e Tecnologia do Rio Grande do Sul (IFRS), Campus Canoas, Canoas, RS, Brasil

$$
\text { Dr. Oclide José Dotto }
$$

Caxias do Sul, RS, Brasil

Dr. Otávio Paulino Lavor

Universidade Federal Rural do Semi-Árido (UFERSA), Campus Pau dos Ferros, Pau dos Ferros, RN, Brasil

Dra. Patrícia Pujol Goulart Carpes

Universidade Federal do Pampa (UNIPAMPA), Itaqui, RS, Brasil 


\section{Dr. Paulo Vilhena da Silva}

Universidade Federal do Pará (UFPA), Instituto de Ciências Exatas e Naturais, Belém, PA, Brasil

Dr. Pedro Lucio Barboza

Universidade Estadual da Paraíba (UEPB), Centro de Ciência e Tecnologia, Departamento de Matemática e Estatística, Campina Grande, PB, Brasil

\section{Dr. Rafael Cavalheiro}

Universidade Federal do Rio Grande (FURG), Campus Santo Antônio da Patrulha, Santo Antônio da Patrulha, RS, Brasil

\section{Dr. Rafael Jorge Pontes Diógenes}

Universidade da Integração Internacional da Lusofonia Afro-Brasileira (UNILAB), Instituto de Ciências Exatas e da Natureza, Campus dos Palmares, Acarape, CE, Brasil

Me. Raimundo Gomes de Souza

Secretaria de Estado de Educação do Pará (SEDUC/PA), Sistema de Organização Modular de Ensino do Pará (SOME/PA), Moju, PA, Brasil

Ma. Rebeca Jéssica Schmitz

Instituto de Educação Tecnológica De Luca Daher (IDD), Curitiba, PR, Brasil

\section{Dr. Rodrigo Nonamor Pereira Mariano de Souza}

Universidade Federal Rural de Pernambuco (UFRPE), Departamento de Estatística e Informática, Recife, PE, Brasil

\section{Dr. Rogério Fernando Pires}

Universidade Federal de Uberlândia (UFU), Faculdade de Ciências Integradas do Pontal, Ituiutaba, MG, Brasil

Dr. Ross Alves do Nascimento

Universidade Federal Rural de Pernambuco (UFRPE), Recife, PE, Brasil

Me. Sidney Lopes Sanchez Júnior

Universidade Federal do Paraná (UFPR), Jandaia do Sul, PR, Brasil

Ma. Simone de Fatima Freitas

Secretaria da Educação do Estado de São Paulo (SEESP), Ourinhos, SP, Brasil

Ma. Terezinha Marisa Ribeiro de Oliveira

Secretaria da Educação do Estado de São Paulo (SEESP), Votorantim, SP, Brasil

Dr. Wagner Marcelo Pommer

Universidade Federal de São Paulo (UNIFESP), Campus Diadema, Diadema, SP, Brasil

Me. Wagner Rodrigues da Silva

Universidade Federal do Rio Grande do Sul (UFRGS), Porto Alegre, RS, Brasil 